\title{
Reducing the Rate of Catheter-Associated Urinary Tract Infection in the Non-ICU Setting
}

\author{
Sameeh Ghazal1*, Syam Clara Viorica1, Mercy Joseph1', Mahmoud Mukahal1, \\ Ahmed Hakawi' ${ }^{1}$, Michael B. Edmond ${ }^{2}$ \\ ${ }^{1}$ King Fahad Medical City, Riyadh, Saudi Arabia \\ ${ }^{2}$ Division of Infectious Diseases, Department of Internal Medicine, University of lowa Carver College of \\ Medicine, lowa City, IA, USA \\ Email: ${ }^{*}$ ghazalkfmc@gmail.com
}

Received 18 May 2015; accepted 17 June 2015; published 23 June 2015

Copyright (C) 2015 by authors and Scientific Research Publishing Inc.

This work is licensed under the Creative Commons Attribution International License (CC BY). http://creativecommons.org/licenses/by/4.0/

(c) (i) Open Access

\begin{abstract}
Background: In 2008, the catheter associated urinary tract infection (CAUTI) rate at King Fahad Medical City (KFMC) was 3.8/1000 urinary catheter days with some variability between departments. KFMC is the newest tertiary, referral and teaching hospital with 1100 beds in Riyadh, Saudi Arabia. The Infection Control Department at KFMC decided to implement a quality improvement project by applying the bladder bundle in our general ward (Non-ICU) using the model of National Health Service (NHS) hospitals in England even though there was good evidence supporting this infection control practice only in ICU patients [1] [2]. Our objective was to decrease CAUTI in two non-ICU units by at least $50 \%$ in one year. Study design: This was a prospective interventional quality improvement project aiming to decrease CAUTI in two non-ICU inpatient units with a total of 193 beds including children and adult patients. Our intervention includes insertion and maintenance components. Results: CAUTI decreased significantly in both departments from 23 infections in 2008 (Rate: 5.03/1000 CDs) to 12 infections in 2009 (Rate: 1.92/1000 CDs) $(P=0.0001)$; in RH (Rehabilitation hospital) from 18 in 2008 (Rate: 4/1000 CDs) to 11 infections in 2009 (Rate: 0.36/1000 CDs) $(P<0.0001)$ and in NSI (National Neuroscience Institute) from 5 in 2008 (Rate: 5.42/1000 CDs) to 1 infections in 2009 (Rate: $3.16 / 1000$ CDs) $(P<0.0001)$. Conclusion: Implementation of urinary catheter insertion and daily care bundles, and creation of a competitive spirit among employees were associated with a significant reduction in catheter associated urinary tract infections.
\end{abstract}

\section{Keywords}

Catheter Associated Urinary Tract Infections, Urinary Tract Infections, CAUTI in Non-ICU Sitting

\footnotetext{
${ }^{*}$ Corresponding author.
}

How to cite this paper: Ghazal, S., Viorica, S.C., Joseph, M., Mukahal, M., Hakawi, A. and Edmond, M.B. (2015) Reducing the Rate of Catheter-Associated Urinary Tract Infection in the Non-ICU Setting. Advances in Infectious Diseases, 5, 81-86. 


\section{Introduction}

In 2008, the catheter associated urinary tract infection (CAUTI) rate at King Fahad Medical City (KFMC) was 3.8/1000 urinary catheter days (85 infections/22,274 catheter days, catheter utilization ratio: 0.13 ) with some variability between departments. CAUTI rates in the National Neuroscience Institute (NNI) and Rehabilitation Hospital (RH), which are component units of KFMC, were 5.4 and 4.0/1000 catheter days, respectively (5.0/1000 in the two departments combined). KFMC is the newest tertiary, referral and teaching hospital with 1100 beds in Riyadh, Saudi Arabia.

The Infection Control Department at KFMC decided to implement a quality improvement project by applying the bladder bundle in our general ward (Non-ICU) using the model of National Health Service (NHS) hospitals in England even though there was good evidence supporting this infection control practice only in ICU patients [1] [2]. Our objective was to decrease CAUTI in two non-ICU units by at least $50 \%$ in one year.

\section{Methods}

\subsection{Study Design}

This was a prospective interventional quality improvement project aiming to decrease CAUTI in two non-ICU inpatient units with a total of 193 beds by 50\% in one year (January - December 2009). Patients admitted to these units were children and adults with neurological and neurosurgical problems, and those who require short course inpatient rehabilitation. The intervention was locally designed bundles modeled after the bundle used in NHS hospitals in England [3], which included insertion and maintenance components.

All patients with urinary catheters inserted and maintained in the two inpatient units were included. Patients excluded if no urinary catheter, or their urinary catheters inserted outside these two units. Monitoring of bundle compliance was discontinued 48 hours after removal of the urinary catheter, or on discharge or transfer from the study units.

\subsection{The Intervention Had Two Components (Bundles)}

A. Urinary catheter insertion bundle, which consists of:

1) Performance of hand hygiene immediately before the procedure;

2) Maintenance of maximum aseptic technique during insertion;

3) Use of a pre-prepared sterile urinary catheter set;

4) Use of the smallest suitable catheter size;

5) Performance of insertion by experienced personnel (previous insertion of more than 5 catheters);

6) Proper securing of the catheter.

B. Urinary catheter maintenance bundle, which consists of:

1) Daily review of the need for the urinary catheter;

2) Hand hygiene before and after manipulating catheters;

3) Maintenance of a closed sterile drainage system;

4) Regular emptying of urinary drainage bags into clean containers;

5) Ensuring that patients are aware of their role in preventing UTI;

6) Daily meatal hygiene with plain soap.

A project team was formed and included by infectious disease specialists (IDs), the hospital epidemiologist, infection preventionists (IPs), and head nurses of the hospital units. An extensive education program was initiated by the team leader for the IPs and head nurses. Patient and family education was performed by the head nurses and IPs.

Several forms were used to collect data: a catheter days form completed by head nurses and reviewed by IPs; an infection monitoring form completed by the IPs and reviewed by an infectious disease specialist; and two checklists for monitoring compliance, one for monitoring compliance with the insertion bundle and another one for monitoring compliance with the maintenance bundle. The compliance checklists were typically completed by the head nurse of each unit. Periodic audits were performed by IPs who completed several competency tests for monitoring insertion and maintenance of the urinary catheters to avoid inter-rater variability. IPs were allowed to complete the checklist only after demonstrating a difference no more than 5\% with a colleague. Collected data was verified and analyzed by the hospital epidemiologist. 
There were no other changes in infection control practice during the study period in both units.

The process measures, including the compliance rates for each item of both bundles as assessed by the IPs, urinary catheter utilization ratio, as well as outcome measures (each unit's CAUTI rate), were reported to each department every three months by the team leader and the results announced in the hospital infection control newsletter.

Throughout the study, concurrent surveillance for CAUTI was performed by trained infection preventionists using the case definition of the National Healthcare Safety Network [4]. The study was approved by our Institutional Review Board.

\subsection{Data Entry and Analysis}

Data was entered and analyzed by the hospital epidemiology department. Statistical analyses included: (1) calculation of the CAUTI rate for each unit before (calendar year 2008) and after the intervention (calendar year 2009); (2) the compliance rate with each component of the insertion bundle; (3) the compliance rate with each component of the daily care bundle.

Case definition and CAUTI rate was following the CDC/NHSN methodology for device-associated infections (dividing the number of catheter-associated urinary tract infections over a time period to the number of urinary catheter-days over the same time period and multiplying by 1000).

The statistical test of the difference in the rates of CAUTI at 95\% confidence level was calculated by the use of Medcalc statistics at http://www.medcalc.org. The $P$-value was considered statistically significant at a level of 0.05 or less.

\section{Results}

CAUTI decreased significantly in both departments from 23 infections in 2008 (Rate: 5.03/1000 CDs) to 12 infections in 2009 (Rate: 1.92/1000 CDs) ( $P=0.0001$ ); in RH from 18 in 2008 (Rate: 4/1000 CDs) to 11 infections in 2009 (Rate: 0.36/1000 CDs) $(P<0.0001)$ and in NS from 5 in 2008 (Rate: 5.42/1000 CDs) to 1 infections in 2009 (Rate: 3.16/1000 CDs) $(P<0.0001)$ (Table 1$)$.

There was a significant reduction in the catheter utilization rate $(0.21$ to $0.19, P$-value: $<0.0001$ with CI: $0.021 \%-0.023 \%)$ in the NS department and there was a significant increase in RH $(0.103$ to $0.137, P$-value: $<0.00001$ with CI: $3.33 \%-3.4 \%$ ), but there was no difference in the catheter utilization rate when both departments were considered together ( 0.16 before and after, $P$-value: $<0.9788$ with CI: $-2.1 \%-2.3 \%)$ (Table 2)

Table 1. Comparison of CAUTI rates before and after the intervention.

\begin{tabular}{cccccccccccc}
\hline & \multicolumn{3}{c}{ Neuroscience department } & \multicolumn{2}{c}{ Rehabilitation department } & \multicolumn{3}{c}{ Total (NEURO + REHAB) } \\
\cline { 2 - 10 } Time Period & CDs & No. Inf & IR & CDs & No. Inf & IR & CDs & No. Inf & IR \\
\hline 1st Q, 2008 & 744 & 5 & 6.72 & 336 & 3 & 8.93 & 1080 & 8 & 7.41 \\
2nd Q, 2008 & 831 & 6 & 7.22 & 330 & 1 & 3.03 & 1161 & 7 & 6.03 \\
3rd Q, 2008 & 897 & 5 & 5.57 & 310 & 1 & 3.23 & 1207 & 6 & 4.97 \\
4th Q, 2008 & 851 & 2 & 2.35 & 275 & 0 & 0.00 & 1126 & 2 & 1.78 \\
Annual, 2008 & 3323 & 18 & 5.42 & 1251 & 5 & 4.00 & 4574 & 23 & 5.03 \\
1st Q, 2009 & 842 & 1 & 1.19 & 747 & 0 & 0.00 & 1589 & 1 & 0.63 \\
2nd Q, 2009 & 964 & 2 & 2.07 & 918 & 0 & 0.00 & 1882 & 2 & 1.06 \\
3rd Q, 2009 & 885 & 3 & 3.39 & 545 & 0 & 0.00 & 1430 & 3 & 2.10 \\
4th Q, 2009 & 795 & 5 & 6.29 & 566 & 1 & 1.77 & 1361 & 6 & 4.41 \\
Annual, 2009 & 3486 & 11 & 3.16 & 2776 & 1 & 0.36 & 6262 & 12 & 1.92
\end{tabular}

CDs = catheter days; No. Inf = number of infections; IR = infection rate (infections/1,000 CDs); UR = catheter utilization rate. 
Compliance with the insertion bundle components was excellent, 98.5\% (Table 3) as assessed by 37 random audits. There were only two episodes where hands were not washed properly before the procedure and the smallest size catheter was not used as recommended.

Due to a shortage of manpower, the checklist for daily urinary catheter maintenance was checked infrequently (17 random audits) and it showed full compliance in 13/17 opportunities (76\%, Table 3).

\section{Discussion}

Urinary tract infections (UTIs) are tied with pneumonia as the second most common type of healthcare-associated infection, second only to surgical site infections (SSIs) [5]. Each year, more than 13,000 deaths are associated with UTIs [6].

UTIs account for more than $15 \%$ of infections reported by acute care hospitals. Virtually all healthcare-associated UTIs are caused by instrumentation of the urinary tract [7]. Guidelines from the Centers for Disease Control (CDC) and the Society for Healthcare Epidemiology of America/Infectious Diseases Society of America describe various interventions for preventing catheter associated UTIs (CAUTIs) in intensive care units (ICUs). [8] [9]. Bundles of interventions are an important strategy, as part of a multimodal approach that focuses efforts on high-yield interventions [10].

Table 2. Urinary catheter utilization rate in both departments.

\begin{tabular}{ccccccccccc}
\hline & \multicolumn{3}{c}{ Neuroscience department } & \multicolumn{3}{c}{ Rehabilitation department } & \multicolumn{2}{c}{ Total (NEURO + REHAB) } \\
\cline { 2 - 10 } Time Period & PDs & CDs & UR & PDs & CDs & UR & PDs & CDs & UR \\
& 3570 & 744 & 0.21 & 3208 & 336 & 0.10 & 6778 & 1080 & 0.16 \\
1st Q, 2008 & 3980 & 831 & 0.21 & 3111 & 330 & 0.11 & 7091 & 1161 & 0.16 \\
2nd Q, 2008 & 4169 & 897 & 0.22 & 2996 & 310 & 0.10 & 7165 & 1207 & 0.17 \\
3rd Q, 2008 & 4049 & 851 & 0.21 & 2807 & 275 & 0.10 & 6856 & 1126 & 0.16 \\
4th Q, 2008 & 15768 & 3323 & 0.21 & 12122 & 1251 & 0.10 & 27890 & 4574 & 0.16 \\
Annual, 2008 & 4478 & 842 & 0.19 & 4980 & 747 & 0.15 & 9458 & 1589 & 0.17 \\
1st Q, 2009 & 4700 & 964 & 0.21 & 5277 & 918 & 0.17 & 9977 & 1882 & 0.19 \\
2nd Q, 2009 & 4713 & 885 & 0.19 & 5022 & 545 & 0.11 & 9735 & 1430 & 0.15 \\
3rd Q, 2009 & 4660 & 795 & 0.17 & 4977 & 566 & 0.11 & 9637 & 1361 & 0.14 \\
4th Q, 2009 & 18551 & 3486 & 0.19 & 20256 & 2776 & 0.14 & 38807 & 6262 & 0.16 \\
Annual, 2009 & & 0.0001 & & & 0.00001 & & & 0.9788
\end{tabular}

CDs = catheter days; PDs = patient days; UR = catheter utilization rate.

Table 3. Compliance with the insertion bundle components as assessed by random audits.

\begin{tabular}{|c|c|c|c|}
\hline & Yes & No & Total \\
\hline Hand hygiene done & 35 & 2 & 37 \\
\hline Sterile gloves used & 37 & 0 & 37 \\
\hline Sterile pack used & 37 & 0 & 37 \\
\hline Smallest catheter size used & 35 & 2 & 37 \\
\hline Trained personnel & 37 & 0 & 37 \\
\hline Catheter secured & 37 & 0 & 37 \\
\hline Full compliance & $98.5 \%$ & $1.5 \%$ & \\
\hline
\end{tabular}


Our study showed that in spite of no significant difference in the utilization ratio of urinary catheters with both departments' data calculated together compared before and after the intervention, there was a significant reduction in the infection rate which strengthened our conclusion that our intervention was effective in reduction of CAUTI in both departments.

Our insertion bundle emphasizes aseptic catheter insertion by mandating proper hand hygiene, using sterile gloves during the procedure, and using a prepackaged, sterile urinary catheter insertion set. The bundle is also designed to minimize trauma via use of the smallest size catheter possible, followed by proper securement, and performed by a skilled operator who can perform the procedure with less trauma and maintain sterility of the procedure.

Preventing bacteria from gaining access to the internal surface of the drainage system or urine is facilitated by always keeping the system closed, avoiding catheter manipulation with unclean hands, and draining the system into a clean container that has not been used for other patients [11].

Our maintenance bundle focuses on shortening the duration of catheterization as much as possible and decreasing the risk of contamination of the drainage system (hand hygiene before manipulation, maintaining a closed system, using a clean container for emptying the drainage system, and ensuring the patient's personal hygiene).

Many studies [12] [13] have emphasized the importance of ongoing surveillance and feedback as an intervention to reduce HAIs. Giving feedback to the staff can create a competitive spirit between departments and staff members to improve their outcomes by being more compliant with the bundle components and more cooperative with infection control staff.

In compliance with CDC guidelines, which recommend [14] that antimicrobial coated catheters should not be used routinely to prevent CAUTI, we did not use these in our hospital. The negative results in a recent randomized controlled trial on the effectiveness of antimicrobial coated urinary catheters [10] supported our decision.

Our result showed a significant decrease in CAUTI for the two units. We believed that implementation of evidence-based recommendations and creation of a competitive spirit between employees had a positive effect on our CAUTI rates.

To keep our success we considered these recommendations as a standard of care for patients with urinary catheter all over the institution.

Our limitations were the small sample size and the inability to continue monitoring our process measures because of a shortage of manpower.

\section{Conclusion}

Implementation of urinary catheter insertion and daily care bundles, and creation of a competitive spirit between employees were associated with a significant reduction in catheter associated urinary tract infections.

\section{Acknowledgements}

None of the author has any conflict of interest. This study was not funded by any individual or organization. IRB of King Fahad Medical City has approved this manuscript.

\section{References}

[1] Jain, M., Miller, L., Belt, D., King, D. and Berwick, D.M. (2006) Decline in ICU Adverse Events, Nosocomial Infections and Cost through a Quality Improvement Initiative Focusing on Teamwork and Culture Change. Qual Saf Health Care, 15, 235-239. http://dx.doi.org/10.1136/qshc.2005.016576

[2] Shuman, E.K. and Chenoweth, C.E. (2010) Recognition and Prevention of Healthcare Associated Urinary Tract Infections in the Intensive Care Unit. Critical Care Medicine, 38, S373-S379. http://dx.doi.org/10.1097/ccm.0b013e3181e6ce8f

[3] Pratt, R.J., Pellowe, C.M., Wilson, J.A., Loveday, H.P., Harper, P.J., Jones, S.R.L.J., et al. (2007) epic2: National Evidence-Based Guidelines for Preventing Healthcare-Associated Infections in NHS Hospitals in England. Journal of Hospital Infection, 65, S1-S64. http://dx.doi.org/10.1016/s0195-6701(07)60002-4

[4] Horan, T.C., Andrus, M., Dudeck, M.A. (2008) CDC/NHSN Surveillance Definition of Health Care-Associated Infection and Criteria for Specific Types of Infections in the Acute Care Setting. American Journal of Infection Control, 36, 309-332. http://dx.doi.org/10.1016/j.ajic.2008.03.002 
[5] http://www.cdc.gov/nhsn/pdfs/pscmanual/7psccauticurrent.pdf

[6] Klevens, R.M., Edward, J.R., Richards Jr., C.L., Horan, T.C., Gaynes, R.P., Pollock, D.A. and Cardo, D.M. (2007) Estimating Health Care-Associated Infections and Deaths in U.S. Hospitals, 2002. Public Health Reports, 122, $160-166$.

[7] Magill, S.S., Hellinger, W., Cohen, J., Kay, R., Bailey, C., Boland, B., et al. (2012) Prevalence of Healthcare-Associated Infections in Acute Care Hospitals in Jacksonville, Florida. Infection Control and Hospital Epidemiology, 33, 283-291. http://dx.doi.org/10.1086/664048

[8] Gould, C.V., Umscheid, C.A., Rajender, K., Agarwal, R.K., Kuntz, G., Pegues, D.A., and the Healthcare Infection Control Practices Advisory Committee (HICPAC) (2009) Guideline for Prevention of Catheter-Associated Urinary Tract Infections. http://www.cdc.gov/hicpac/cauti/001_cauti.html

[9] Lo, E., Nicolle, L., Classen, D., Arias, K.M., Podgorny, K., Anderson, D.J., et al. (2008) Strategies to Prevent Catheter-Associated Urinary Tract Infections in Acute Care Hospitals. Infection Control and Hospital Epidemiology, 29, S41-S50. http://dx.doi.org/10.1086/591066

[10] Pickard, R., Lam, T., Maclennan, G., Starr, K., Kilonzo, M., McPherson, G., et al. (2012) Antimicrobial Catheters for Reduction of Symptomatic Urinary Tract Infection in Adults Requiring Short-Term Catheterization in Hospital: A Multicenter Randomized Controlled Trial. The Lancet, 380, 1927-1935. http://dx.doi.org/10.1016/S0140-6736(12)61380-4

[11] Apisarnthanarak, A., Thongphubeth, K., Sirinvaravong, S., Kitkangvan, D., Yuekyen, C., Warachan, B., et al. (2007) Effectiveness of Multifaceted Hospital Wide Quality Improvement Programs Featuring an Intervention to Remove Unnecessary Urinary Catheters at a Tertiary Care Center in Thailand. Infection Control and Hospital Epidemiology, 28, 791-798. http://dx.doi.org/10.1086/518453

[12] Fakih, M.G., Watson, S.R., Greene, M.T., Kennedy, E.H., Olmsted, R.N., Krein, S.L. and Saint, S. (2012) Reducing Inappropriate Urinary Catheter Use: A Statewide Effort. Archives of Internal Medicine, 172, 255-260. http://dx.doi.org/10.1001/archinternmed.2011.627

[13] Dumigan, D.G., Kohan, C.A., Reed, C.R., Jekel, J.F. and Fikrig, M.K. (1998) Utilizing National Nosocomial Infection Surveillance System Data to Improve Urinary Tract Infection Rates in Three Intensive-Care Units. Clinical Performance and Quality Healthcare, 6, 172-178.

[14] Gould, C.V., Umscheid, C.A., Agarwal, R.K., Kuntz, G., Pegues, D.A., and Healthcare Infection Control Practices Advisory Committee (HICPAC) (2010) Guideline for Prevention of Catheter-Associated Urinary Tract Infections 2009. Infection Control and Hospital Epidemiology, 31, 319-326. http://dx.doi.org/10.1086/651091

\author{
Abbreviations \\ CAUTI: Catheter associated urinary tract infections; \\ KFMC: King Fahad Medical City; \\ ICU: Intensive care unit; \\ Non-ICU: Non intensive care unit; \\ RH: Rehabilitation hospital; \\ NSI: National neuroscience institute; \\ CDs: Catheter days; \\ IPs: Infection preventionists;
}

